\title{
Large current density from carbon nanotube field emitters
}

\author{
W. Zhu ${ }^{\text {a) }}$ \\ Bell Laboratories, Lucent Technologies, Murray Hill, New Jersey 07974
}

C. Bower and O. Zhou

Department of Physics and Astronomy, University of North Carolina, Chapel Hill, North Carolina 27599

G. Kochanski and S. Jin

Bell Laboratories, Lucent Technologies, Murray Hill, New Jersey 07974

(Received 3 March 1999; accepted for publication 16 June 1999)

\begin{abstract}
We observe that field emitters made from carbon nanotubes exhibit excellent macroscopic emission properties; they can operate at a very large current density, as high as $4 \mathrm{~A} / \mathrm{cm}^{2}$. At electric fields as low as $4-7 \mathrm{~V} / \mu \mathrm{m}$, they emit technologically useful current densities of $10 \mathrm{~mA} / \mathrm{cm}^{2}$. We show that the emission originates from nanotube ends with a characteristic structured ring pattern. The emission characteristics and durability of the carbon nanotube cold cathodes offer promising applications for vacuum microelectronic devices. (C) 1999 American Institute of Physics.

[S0003-6951(99)02332-3]
\end{abstract}

Carbon nanotubes are a stable form of carbon and can now be fabricated by several techniques. ${ }^{1-3}$ They are typically made as threads about $10-30 \mathrm{~nm}$ in diameter with a high aspect ratio $(>1000)$. In our experiments, each thread is a bundle of single-wall nanotubes (SWNT). These geometric properties, coupled with their high mechanical strength and chemical stability, make carbon nanotubes attractive as electron field emitters. Several groups have recently reported observations of electron field emission from nanotubes. ${ }^{4-9}$ However, studies of the emission mechanism are lacking, and the reported current densities are rather low, typically, $0.1-100 \mathrm{~mA} / \mathrm{cm}^{2}$ [reported emission at $400 \mathrm{~mA} / \mathrm{cm}^{2}$ (Ref. $10)$ is hard to interpret, as the data are unexpectedly independent of anode-cathode distance].

In this letter, we report the observation of emission from individual nanotubes, with large macroscopically averaged current densities emitted by a deposit of SWNTs on a substrate. Individual nanotubes show one of two patterns of emission. The first is emission along the axis of the tube, and the other is a characteristic ring pattern where the electrons are emitted with substantial perpendicular momentum $\left(k_{\perp}\right)$. These two patterns presumably originate from structural differences by way of their different Fermi surfaces. ${ }^{11,12} \mathrm{Col}-$ lectively, the nanotubes emit a large current density, which routinely exceeds $1 \mathrm{~A} / \mathrm{cm}^{2}$, and can exceed $4 \mathrm{~A} / \mathrm{cm}^{2}$. Such sustained, high-current field emission is necessary for many technological applications: for example, flat-panel fieldemission displays require $10 \mathrm{~mA} / \mathrm{cm}^{2}$, while microwave power amplifier tubes require at least $500 \mathrm{~mA} / \mathrm{cm}^{2}$.

These high-curent density levels were previously obtainable only by thermionic emission above $1000 \mathrm{~K},{ }^{13}$ or lithographically fabricated tips. ${ }^{14}$ Field emission has been reported from other forms of carbon, however, at lower current densities $\left[0.3 \mathrm{~mA} / \mathrm{cm}^{2}\right.$ for graphite, ${ }^{15} 30 \mathrm{~mA} / \mathrm{cm}^{2}$ for diamond (Refs. 16 and 17, and references therein)]. While higher current densities can be deduced if localized emission is assumed [e.g., $100 \mathrm{~A} / \mathrm{cm}^{2}$ (Refs. 16)], the actual areas are

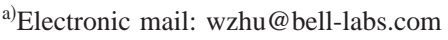

not measured, and the localized emission hot spots are neither understood nor reproducible.

Our emitters are SWNT films deposited on Si substrates. They are grown in a laser ablation system, as described previously. ${ }^{18}$ The as-deposited raw material contains about $70 \%$ in volume [by transmission electron microscopy (TEM), scanning electron microscopy (SEM), and Raman measurements] of SWNTs with an average tube diameter of $1.3-1.6 \mathrm{~nm}$ in $10-30 \mathrm{~nm}$ bundles. Figure 1(a) is a highresolution transmission electron micrograph of the SWNTs.

(a)

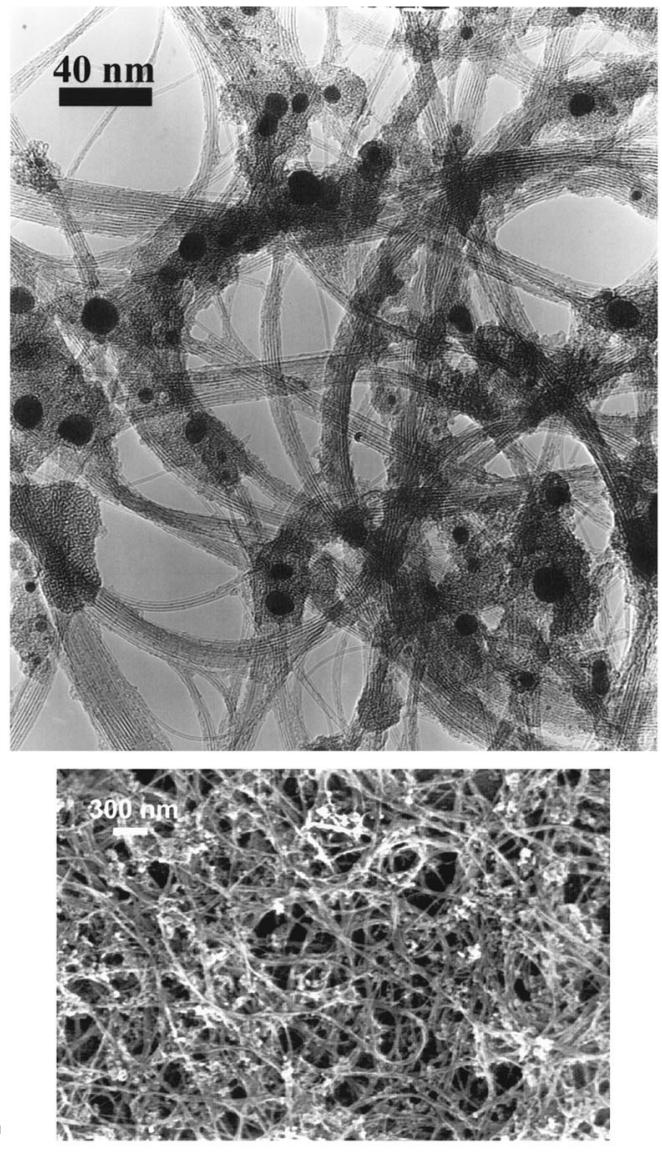

FIG. 1. (a) TEM micrograph showing the bundled nature of the nanotubes, and (b) SEM micrograph of emitters. 


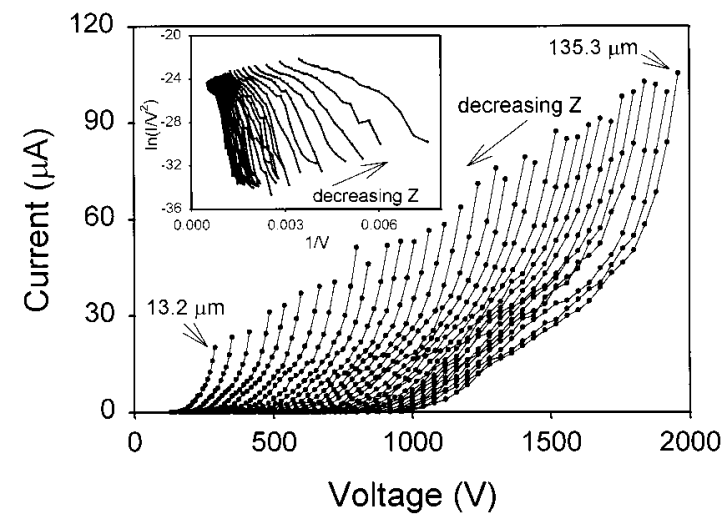

FIG. 2. Emission $I-V$ data. The inset is a $\log \left(I / V^{2}\right)$ vs $1 / V$ plot.

Emitters are made of purified SWNTs by ultrasonically dispersing the nanotubes in a solvent, filtering, and redepositing on a Si substrate. Figure 1(b) shows a scanning electron micrograph of such a SWNT emitter. The emitter contains a high density of nanotubes on the surface. Each of the curved lines in the micrograph represents a SWNT bundle.

The field-emission measurements were carried out at room temperature in a vacuum chamber with a $10^{-8}$ Torr base pressure. The experimental procedures for current density measurements were described in detail elsewhere. ${ }^{19}$ Briefly, a voltage up to $2 \mathrm{kV}$ was applied to a hemispherical molybdenum anode probe (radius of curvature $R \approx 250 \mu \mathrm{m}$ ). A translation stage in the vacuum chamber was used to control the distance between the anode and the cathode, typically varying in the range of $10-300 \mu \mathrm{m}$. The emission current-voltage $(I-V)$ characteristics were measured as a function of the anode-cathode distance $(Z)$. At each distance, the anode voltage was raised from zero until the current density reached $\sim 0.6 \mathrm{~A} / \mathrm{cm}^{2}$ and then decreased back to zero. The effective emission area (A) used to calculate the current density is the area within which $J>1 / 2 J_{\max }$, where $J_{\max }$ is the emission current density directly beneath the anode where the electric field is the highest. For $Z \ll 2 R, A$ $=2 \pi R Z\left(2^{1 / n}-1\right)$, where $n=(V / I)(d I / d V)$. Typically, $n$ $\sim 18$ for $J<50 \mathrm{~mA} / \mathrm{cm}^{2}$, decreasing gradually as the current density increases.

The anode was then moved one step $(3.3 \mu \mathrm{m})$ closer to the emitter surface, and the cycle was repeated. To determine the anode-cathode distance, the capacitance between the anode and the emitter was measured and fitted. ${ }^{19}$ Nanotubes are flexible enough to bend and align themselves with the applied field. In fact, the symmetry of the emission patterns, as discussed below, implies that all loose ends are aligned with the electric field.

Figure 2 shows the measured emission current $I$ as a function of applied voltage $V$. Smooth and consistent $I-V$ curves were measured. In the inset, the data are also shown as $\log \left(I / V^{2}\right)$ vs $1 / V$; in those coordinates, data that follow the Fowler-Nordheim equation ${ }^{20}$ would fall on a straight line. However, the Fowler-Nordheim equation was derived for planar surfaces and is not strictly valid for SWNTs, since the radius of the nanotubes is comparable to the tunneling barrier. ${ }^{21}$ The space-charge limit at our applied electric field is an order of magnitude higher than our largest current density, so we expect it to have little effect. ${ }^{22,23}$ The turn-on field, for an emission current of $1 \mathrm{nA}$, is 1.7

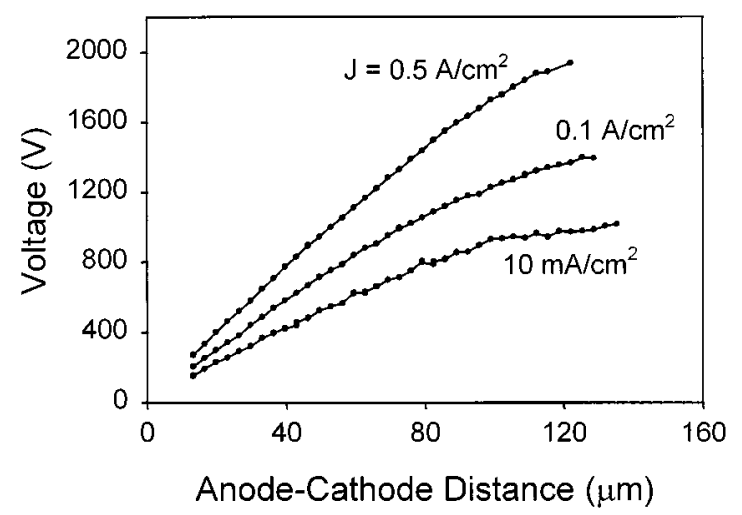

FIG. 3. Voltage vs anode-cathode distance at various current densities. The $x$ axis is determined by capacitance measurements.

$\mathrm{V} / \mu \mathrm{m}$. The threshold field, which we define as the field required to generate an emission current density of 10 $\mathrm{mA} / \mathrm{cm}^{2}$, is $6.5 \mathrm{~V} / \mu \mathrm{m}$ for this sample, as shown in Fig. 3. Also shown are voltages required to produce larger current densities of 0.1 and $0.5 \mathrm{~A} / \mathrm{cm}^{2}$. The slight change in slope as the anode-cathode distance is gradually reduced may be attributable to the initial conditioning or burn-in of the emitter. Reproducibility tests from different locations of the same sample and from other similar SWNT mats and films consistently yield threshold fields of $4-7 \mathrm{~V} / \mu \mathrm{m}$.

In order to identify the origin of the emission sites, we placed a phosphor screen $500 \mu \mathrm{m}$ above the emitter at a potential of $700 \mathrm{~V}$. Surprisingly, the emission is structured as shown in Fig. 4, in which well-defined rings of electron emission, rather than the speckled pattern one sees with other emitters (e.g., diamond), are seen. Individual rings appear and disappear as a unit, implying that they correspond to single microscopic emission sites. Because of the circular symmetry of the rings, they must be emission from the end of nanotubes, rather than the sides of loops of nanotube, which would yield a twofold symmetric pattern. The rings on the phosphor screen are large $(\sim 150 \mu \mathrm{m})$, far larger than any structural features in the sample; therefore, they must be images of the momentum distribution of electrons leaving individual emission sites.

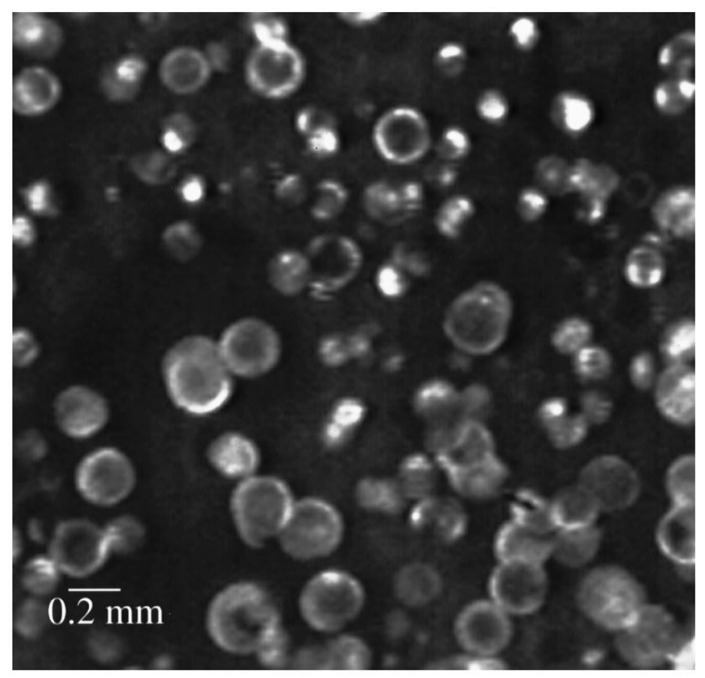

FIG. 4. Structured emission pattern from nanotube ends projected on a phosphor screen. Rings and circles of emission may result from two different types of nanotubes.

ent types of nanotubes.
AIP license or copyright, see http://apl.aip.org/apl/copyright.jsp 


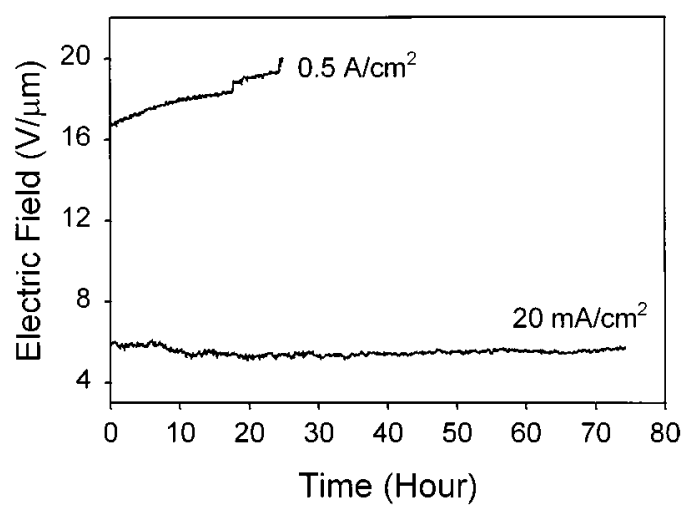

FIG. 5. Electric field required to maintain constant emission current density over time.

The emission site density is estimated to be about $1000 / \mathrm{cm}^{2}$ at the low field of $1.5 \mathrm{~V} / \mu \mathrm{m}$ applied between the phosphor and the nanotube emitter. The emission site density is seen to increase as higher fields are applied. However, we are unable to accurately determine the site density at high current conditions because the phosphor becomes overloaded. We note that loops of nanotube might also contribute to emission at large electric fields, where the current density was too large for us to observe emission patterns.

The nanotube emitters studied here are capable of generating very high emission current densities. As we increase the voltage, the emission current smoothly increases to 0.4 $\mathrm{mA}$ at $3 \mathrm{kV}$. This corresponds to a current density of 4 $\mathrm{A} / \mathrm{cm}^{2}$, at a conservatively large estimate of the anodecathode gap, $50 \mu \mathrm{m}$, and a measured $n,(V / I) d I / d V$, of 6 . This is a very high emission current density reported for carbon nanotube emitters, and is at least one order of magnitude higher than previously reported values. The primary uncertainty is from the thermal expansion of the anode due to electron-impact heating, which tends to close the gap. The current density could be as high as $10 \mathrm{~A} / \mathrm{cm}^{2}$, if one uses the best estimate of the anode's thermal expansion.

We have achieved higher currents, and higher current densities (e.g., $0.7 \mathrm{~mA}$ at $4 \mathrm{kV}$ ), but under these extreme conditions, the thermal expansion of the anode makes the size of the anode-cathode gap too uncertain to accurately calculate field or current density values. Due to the exceptionally high emission current impinging on the Mo anode probe, it gradually glows red and then yellow. Modeling of the thermal transport in the anode yields a probe tip temperature of $1980 \mathrm{~K}$ at the highest currents, consistent with visual observations. Emitters rarely failed due to electrical overload. Thermal enhancement of the field emission is still relatively small, because the emitter temperature is low compared to the work function. ${ }^{24}$

Upon operating at the emission current density of 4 $\mathrm{A} / \mathrm{cm}^{2}$, the structure of the nanotube cathode remains intact, as confirmed by SEM examination after the emission measurements. We attribute the excellent stability to the structural perfection of the nanotubes fabricated by laser ablation, ${ }^{3,18}$ and the well-controlled emitter fabrication procedure. The durability of nanotube emitters was tested by measuring the voltage needed to maintain a constant current density. As shown in Fig. 5, at the current density of 20 $\mathrm{mA} / \mathrm{cm}^{2}(10 \mu \mathrm{A}$ actual current at $Z \approx 250 \mu \mathrm{m})$, the field re- mained constant at $5.5 \mathrm{~V} / \mu \mathrm{m}$ for a period of $75 \mathrm{~h}$. At the current density of $0.5 \mathrm{~A} / \mathrm{cm}^{2}(100 \mu \mathrm{A}$ actual current at $Z$ $\approx 100 \mu \mathrm{m}$ ), the required field increased slowly, from about 16.5 to $20 \mathrm{~V} / \mu \mathrm{m}$ over $25 \mathrm{~h}$. This gradual damage may be attributable to ion bombardment from the background gas in the vacuum chamber. Improved stability is likely if the nanotube emitters are operated at higher vacuum, e.g., above $10^{-8}$ Torr.

We note that the ultimate limits to the current density obtainable from nanotube emitters are far higher than observed here. Since individual nanotubes can stably emit 30 nA (our total phosphor screen current divided by the number of emission spots), one could envision average current densities reaching as high as $10^{5}-10^{7} \mathrm{~A} / \mathrm{cm}^{2}$, from an array of tubes on a $0.1-1 \mu \mathrm{m}$ lattice.

In summary, we have established that electron emission from nanotubes originates from the ends of the tubes. The nanotube emitters exhibit excellent macroscopic emission properties. The emission characteristics and durability of the carbon nanotube cold cathodes offer promising applications for vacuum microelectronic devices.

The authors acknowledge valuable discussions with C. M. Varma and L. Balents from Bell Labs. The research work carried out at UNC is supported by the Office of Naval Research. The fellowship support for one of the authors (C.B.) from NASA is also acknowledged.

${ }^{1}$ S. Iijima, Nature (London) 354, 56 (1991).

${ }^{2}$ T. W. Ebbesen and P. M. Ajayan, Nature (London) 358, 220 (1992).

${ }^{3}$ B. I. Yakobson and R. E. Smalley, Am. Sci. 85, 324 (1997).

${ }^{4}$ A. G. Rinzler, J. H. Hafner, P. Nikolaev, L. Lou, S. G. Kim, D. Tomanek, P. Nordlander, D. T. Colbert, and R. E. Smalley, Science 269, 1550 (1995).

${ }^{5}$ W. A. de Heer, A. Chatelain, and D. Ugarte, Science 270, 1179 (1995).

${ }^{6}$ Q. H. Wang, T. D. Corrigan, J. Y. Dai, R. P. H. Chang, and A. R. Krauss, Appl. Phys. Lett. 70, 3308 (1997).

${ }^{7}$ Y. Saito, S. Uemura, and K. Hamaguchi, Jpn. J. Appl. Phys., Part 2 37, L346 (1998).

${ }^{8}$ Q. H. Wang, A. A. Setlur, J. M. Lauerhaas, J. Y. Dai, E. W. Seelig, and R. P. H. Chang, Appl. Phys. Lett. 72, 2912 (1998).

${ }^{9}$ J. M. Bonard, J. P. Salvetat, T. Stockli, W. A. de Heer, L. Forro, and A. Chatelain, Appl. Phys. Lett. 73, 918 (1998).

${ }^{10}$ P. G. Collins and A. Zettl, Appl. Phys. Lett. 69, 1969 (1996).

${ }^{11}$ M. S. Dresselhaus, G. Dresselhaus, and P. C. Ecklund, Science of Fullerenes and Carbon Nanotubes (Academic, New York, 1996), p. 756ff.

${ }^{12}$ Carbon Nanotubes, edited by T. W. Ebbesen (CRC, Cleveland, OH, 1997), pp. 191-210.

${ }^{13}$ G. Hermann and S. Wagener, The Oxide Coated Cathode (Chapman and Hall, London, 1951).

${ }^{14}$ I. Brodie and C. A. Spindt, in Adv. Electron. Electron Phys., edited by P. W. Hawkes (Academic, New York, 1992), Vol. 83, p. 1.

${ }^{15}$ S. Bajic and R. V. Latham, J. Phys. D 21, 200 (1988).

${ }^{16}$ K. Okano, T. Yamada, H. Ishihara, F. Koizumi, and J. Itoh, Appl. Phys. Lett. 70, 2201 (1997).

${ }^{17}$ W. Zhu, G. P. Kochanski, and S. Jin, Science 282, 1471 (1998).

${ }^{18}$ C. Bower, S. Suzuki, K. Tanigaki, and O. Zhou, Appl. Phys. A: Mater. Sci. Process. 67A, 47 (1998).

${ }^{19}$ W. Zhu, G. P. Kochanski, and S. Jin, J. Vac. Sci. Technol. B 14, 2011 (1996).

${ }^{20}$ R. H. Fowler and L. W. Nordheim, Proc. R. Soc. London, Ser. A 119, 173 (1928).

${ }^{21}$ J. He, P. H. Cutler, N. M. Miskovsky, T. E. Feuchtwang, T. E. Sullivan, and M. Chung, Surf. Sci. 246, 348 (1991).

${ }^{22}$ J. P. Barbour, W. W. Dolan, J. K. Trolan, E. E. Martin, and W. P. Dyke, Phys. Rev. 92, 45 (1953).

${ }^{23}$ A. E. Bell and L. W. Swanson, Phys. Rev. B 19, 3353 (1979).

${ }^{24}$ R. H. Good, Jr. and E. W. Mueller, in Handbuch der Physik, edited by S. Fluace (Springer, Berlin, 1956), Vol. 21, pp. 176-231. 